\title{
Static and dynamic fracture analysis in thin piezoelectric laminas by the boundary element method
}

\author{
H. Y. Wang ${ }^{1}$, J. Lei ${ }^{1} \&$ C. H. Zhang ${ }^{2}$ \\ ${ }^{I}$ Department of Engineering Mechanics, \\ Beijing University of Technology, China \\ ${ }^{2}$ Department of Civil Engineering, University of Siegen, Germany
}

\begin{abstract}
A boundary element method (BEM) is developed to study the static and dynamic fracture problems in a thin piezoelectric structure. The method uses quadrature formula and the collocation method for the temporal and spatial discretization. A quadratic quarter-point element is implemented at the crack tip. The strongly singular and hypersingular integrals are evaluated by a regularization technique based on a suitable variable change. The nearly singular integrals arisen in thin structures are dealt with in two ways. One is based on a nonlinear coordinate transformation for curve-quadratic element, and the other is based on an analytical integration method for straight quadratic element. A displacement extrapolation technique is used to determine the intensity factors (IFs) including the stress intensity factors (SIFs) and electrical displacement intensity factor (EDIF). Numerical examples are presented to verify the effectiveness and stability of present BEM in a thin piezoelectric structure.

Keywords: thin piezoelectric structure, boundary element method, nearly singular integration, stress intensity factors, electrical displacement intensity factor.
\end{abstract}

\section{Introduction}

With intrinsic electro-mechanical coupling property, piezoelectric material is widely used in various smart structures. Some typical piezoelectric materials, such as PZT and PVDF, are difficultly applied to the structures of complex shape. Therefore, painting technology is developed, and piezoelectric coatings 
are formed on the structure. However, cracks may occur in the coating or between the coating and the matrix under complex coupled stress/electrical loadings. So it's significant to study the fracture problem in the thin piezoelectric structure.

For a general dynamic crack problem in piezoelectric material, numerical methods are more feasible due to the mathematical complexity of the initial boundary value problems. Particularly, BEM has certain advantages in fracture analysis. In 2008, time-domain BEM for transient dynamic crack analysis of linear piezoelectric solid was implemented by García-Sánchez et al. [1], who used the Lubich convolution quadrature formula for the temporal discretization and a collocation method for the spatial discretization. However, its extension to dynamic cracks in thin piezoelectric structure is not straight-forward, since the corresponding dynamic Green's functions have quite complicated mathematical structure, which generates nearly singularity during integration when the field point is very close to the source point. In 2002, Liu and Fan [2] successfully used BEM in the static analysis of thin piezoelectric solid. The nearly singular integrals are solved by an analytical method. But they didn't take cracks in structure into consideration.

To apply the time-domain BEM to the crack analysis of thin piezoelectric structure, two methods are developed to deal with the nearly singular integrals. The first one is based on a nonlinear coordinate transformation method for curve-quadratic element. The second method is based on an analytical integration method for straight quadratic element. Numerical results are presented to verify the accuracy of the present integral equations and the timedomain BEM.

\section{Time-domain BIEs for piezoelectric materials}

\subsection{Piezoelectric equations}

Consider a 2D homogeneous, linear cracked piezoelectric solid occupying domain $\Omega$ with boundary $\Gamma$. To describe the electric-elastic fields, the 'extended' variables $u_{J}, f_{J}, \sigma_{i J}, \varepsilon_{J i}$ are defined

$$
\begin{aligned}
& u_{J}=\left\{\begin{array}{ll}
u_{j} \\
\phi
\end{array}, \quad \sigma_{i J}= \begin{cases}\sigma_{i j} \\
D_{i}\end{cases} \right. \\
& f_{J}= \begin{cases}f_{j} & J=j=1,2 \\
-q & J=3\end{cases} \\
& \varepsilon_{J i}= \begin{cases}\varepsilon_{j i} & J=j=1,2 \\
E_{i} & J=3\end{cases}
\end{aligned}
$$

which combine the elastic variables involving the displacement $u_{j}$, body force $f_{j}$, stress $\sigma_{i j}$, strain $\varepsilon_{i j}$, and the electric ones including electric potential $\phi$, charge $q$, electrical displacement $D_{j}$, field $E_{j}$. Without body forces and electrical charge, 
the governing equations and the constitutive equations under quasi-electrostatic assumption are given by

$$
\sigma_{i J, i}=\rho \delta_{J K}^{*} \ddot{u}_{K}, \quad \sigma_{i J}=C_{i J K l} \varepsilon_{K l}
$$

where $\rho$ is the mass density, "," designates spatial derivative, while ".." denotes temporal derivative. The capital index is from 1 to 3 while lower case letter index takes 1 or 2 . The extended Kronecker delta $\delta_{J K}^{*}$ is defined by

$$
\delta_{J K}^{*}= \begin{cases}\delta_{J K} & J, K=1,2 \\ 0 & \text { otherwise }\end{cases}
$$

Material constants $C_{i J K l}$ are as follows

$$
C_{i J K l}=\left\{\begin{array}{lll}
c_{i j k l}, & J=j=1,2 ; & K=k=1,2 \\
e_{j i l}, & K=3 ; & J=j=1,2 \\
e_{k l i}, & J=3 ; & K=k=1,2 \\
-\kappa_{i l}, & J=K=3 &
\end{array}\right.
$$

in which $c_{i j k l}, e_{i j k}$ and $\kappa_{i k}$ are the elasticity constants, piezoelectric constants and dielectric constants, respectively. The extended strain and displacement relations are given by

$$
\varepsilon_{i j}=\left(u_{i, j}+u_{j, i}\right) / 2, E_{i}=-\varphi_{, i}
$$

\subsection{Time-domain boundary integral equations}

On the boundary, the displacement and traction BIEs are

$$
\begin{aligned}
c_{I J}\left(\boldsymbol{x}_{0}\right) u_{J}\left(\boldsymbol{x}_{0}, t\right)=\int_{\Gamma}\left(u_{I J}^{\mathrm{G}} * p_{J}-p_{I J}^{\mathrm{G}} * u_{J}\right) \mathrm{d} \Gamma \\
\quad+\int_{\Gamma_{C}^{+}} p_{I J}^{\mathrm{G}} * \Delta u_{J} \mathrm{~d} \Gamma, \boldsymbol{x}_{0} \in \Gamma . \\
p_{I}\left(\boldsymbol{x}_{0}, t\right)=\int_{\Gamma}\left(d_{I J}^{\mathrm{G}} * p_{J}-s_{I J}^{\mathrm{G}} * u_{J}\right) \mathrm{d} \Gamma \\
+\int_{\Gamma_{C}^{+}} s_{I J}^{\mathrm{G}} * \Delta u_{J} \mathrm{~d} \Gamma, \boldsymbol{x}_{0} \in \Gamma_{C}^{+},
\end{aligned}
$$

where $c_{I J}(\mathrm{x})$ are free term constants, “*”denotes Riemann convolution, $\Delta u_{J}$ are the extended crack-opening-displacements, $u_{I J}^{G}, p_{I J}^{G}, d_{I J}^{G}$ and $s_{I J}^{G}$ are fundamental solutions derived by Wang and Zhang [3]. $p_{I J}^{G}$ and $d_{I J}^{G}$ have a strong singularity of Cauchy type, while $s_{I J}^{G}$ has a hypersingularity. To deal with the singularity, the fundamental solutions are separated into a singular static and a regular dynamic part. 


\subsection{Laplace-domain dynamic fundamental solutions}

The traction fundamental solutions $p_{I J}^{G}$ and $d_{I J}^{G}$ have a strong singularity of Cauchy type, while the higher-order traction fundamental solution $s_{I J}^{G}$ has a hypersingularity. To deal with the singularity, it is advantageous to split the fundamental solutions into a singular static part and a regular dynamic part. The singular static parts are given by

$$
\begin{gathered}
p_{I J}^{\mathrm{G}(\mathrm{S})}\left(\boldsymbol{x}_{0}, \boldsymbol{x}\right)=\frac{1}{\pi} \operatorname{Re}\left[\sum_{M=1}^{3} L_{J M} Q_{M I} \frac{\mu_{M} n_{1}(\boldsymbol{x})-n_{2}(\boldsymbol{x})}{z_{M}-z_{M}^{0}}\right] \\
d_{I J}^{\mathrm{G}(\mathrm{S})}\left(\boldsymbol{x}_{0}, \boldsymbol{x}\right)=-\frac{1}{\pi} \operatorname{Re}\left[\sum_{M=1}^{3} L_{J M} Q_{M I} \frac{\mu_{M} n_{1}\left(\boldsymbol{x}_{0}\right)-n_{2}\left(\boldsymbol{x}_{0}\right)}{z_{M}-z_{M}^{0}}\right] \\
S_{I J}^{\mathrm{G}(\mathrm{S})}\left(\boldsymbol{x}_{0}, \boldsymbol{x}\right)=\frac{1}{\pi} \operatorname{Re}\left[\sum_{M=1}^{3} T_{I J}^{M} \frac{\mu_{M} n_{1}(\boldsymbol{x})-n_{2}(\boldsymbol{x})}{\left(z_{M}-z_{M}^{0}\right)^{2}}\right]
\end{gathered}
$$

where

$$
z_{M}=x+\mu_{M} y, \quad z_{M}^{0}=x_{0}+\mu_{M} y_{0}, \quad M=1,2,3
$$

$\boldsymbol{x}_{0}\left(x_{0}, y_{0}\right)$ is the source point while $\boldsymbol{x}(x, y)$ the observation point. $L_{J M}, Q_{M I}$, $T_{I J}^{M}$ and $\mu_{M}$ are determined by the anisotropic material constants [3].

\subsection{Time-stepping scheme}

To approximate the Riemann convolution integrals in BIEs, Lubich quadrature formula is used [1]

$$
f(t)=g(t) * h(t) \cong \sum_{j=0}^{n} \omega_{n-j}(\Delta t) h(j \Delta t)
$$

where time $t$ is divided into $N$ equal time-steps, and the weights $\omega_{n-j}(\Delta t)$ are determined by

$$
\omega_{n-j}(\Delta t)=\frac{r^{-(n-j)}}{N} \sum_{m=0}^{N-1} \hat{g}\left[\delta\left(\zeta_{m}\right) / \Delta t\right] \mathrm{e}^{-2 \pi i \cdot(n-j) m / N}
$$

in which $\hat{g}(\cdot)$ stands for the Laplace-transformation of the function $g(t)$. After the temporal and spatial discretization, a system of linear equations for the discrete boundary quantities can be obtained. Leaving all the unknown boundary quantities on the left-hand side, an explicit time-stepping scheme

$$
\boldsymbol{x}_{k}^{n}=\left(\mathbf{A}_{k l}^{0}\right)^{-1} \cdot\left(\boldsymbol{y}_{l}^{n}-\sum_{j=1}^{n-1} \mathbf{A}_{k l}^{n-j} \cdot \boldsymbol{x}_{l}^{j}\right)
$$

can be obtained for computing the unknown boundary quantities at the $n$-th timestep. 


\section{Computation of nearly singular integrals}

When the collocation point is very close to the integration point in thin piezoelectric structure, nearly singular integrals appear. To solve the problem, two methods are developed for curve quadratic elements and straight quadratic element. The nearly singular integrals can be classified into three kinds

$$
\begin{aligned}
& J_{M}=\int_{\Gamma_{e}} \frac{\mu_{M} n_{1}(\boldsymbol{x})-n_{2}(\boldsymbol{x})}{z_{M}-z_{M}^{0}} \phi_{q} d \Gamma, \\
& J_{M}^{\prime}=\int_{\Gamma_{e}} \frac{\mu_{M} n_{1}(\boldsymbol{x})-n_{2}(\boldsymbol{x})}{\left(z_{M}-z_{M}^{0}\right)^{2}} \phi_{q} d \Gamma, \\
& J_{M}^{\prime \prime}=\int_{\Gamma_{e}} \frac{\mu_{M} n_{1}\left(\boldsymbol{x}_{0}\right)-n_{2}\left(\boldsymbol{x}_{0}\right)}{z_{M}-z_{M}^{0}} \phi_{q} d \Gamma .
\end{aligned}
$$

where

$$
z_{M}=x+\mu_{M} y, z_{M}^{0}=x_{0}+\mu_{M} y_{0}, M=1,2,3
$$

where $\left(x_{0}, y_{0}\right)$ is the source point while $(x, y)$ the observation point.

\subsection{Curve quadratic element}

For isoparametric quadratic element, local coordinate system $\xi(\xi \in[-1,1])$ is introduced. When collocation point $x_{0}$ is very close to $\Gamma_{e}$, the nearest point on $\Gamma_{e}$ to $x_{0}$ is assumed to be $\mathrm{Q}\left(x^{\prime}, y^{\prime}\right)$ which has the local coordinate $\xi^{\prime}$, so $z_{M}^{\prime}=x^{\prime}+\mu_{M} y^{\prime}$.

Let $\chi_{M}=z_{M}-z_{M}^{\prime}, \chi_{M}^{0}=z_{M}^{\prime}-z_{M}^{0}, J_{M}$ can be transformed into

$$
J_{M}=\int_{\Gamma_{e}}\left[1 /\left(\chi_{M}+\chi_{M}^{0}\right)\right] \phi_{q} d \chi_{M}
$$

With the substitution of $\phi_{q}$ Taylor expansion, $J_{M}$ can be separated into two parts as follows

$$
\begin{aligned}
J_{M}=\int_{\Gamma_{e}} & {\left[\left(\phi_{q}-\phi_{q 0}\right) /\left(\chi_{M}+\chi_{M}^{0}\right)\right] d \chi_{M} } \\
& +\phi_{q 0} \int_{\Gamma_{e}}\left[1 /\left(\chi_{M}+\chi_{M}^{0}\right)\right] d \chi_{M} .
\end{aligned}
$$

The second integral can be calculated analytically as

$$
\int_{\Gamma_{e}}\left[1 /\left(\chi_{M}+\chi_{M}^{0}\right)\right] d \chi_{M}=\left.\log \left(\chi_{M}+\chi_{M}^{0}\right)\right|_{\Gamma_{e}} .
$$

For the first integral in eqn (6), with the substitution of local coordinate, the detailed formulation for $q=1$ is 


$$
\begin{aligned}
& \int_{\Gamma_{e}} \frac{\phi_{1}-\phi_{10}}{\chi_{M}+\chi_{M}^{0}} d \chi_{M} \\
& \quad=\int_{\Gamma_{e}} \frac{\left[\mu_{M}(c \xi+d)+(a \xi+b)\right]\left[\left(\xi-\xi^{\prime}\right)^{2}+\left(\xi-\xi^{\prime}\right)\left(2 \xi^{\prime}-\xi_{3}\right)\right]}{\left(\xi_{1}-\xi_{3}\right) \xi_{1}\left[a_{M}\left(\xi-\xi^{\prime}\right)^{2}+b_{M}\left(\xi-\xi^{\prime}\right)+c_{M}\right]} d \xi
\end{aligned}
$$

where

$$
\begin{aligned}
& a_{M}=\frac{a+\mu_{M} c}{2}, \\
& b_{M}=2 a_{M} \xi^{\prime}+\left(b+\mu_{M} d\right), \\
& c_{M}=a_{M} \xi^{\prime 2}+\left(b+\mu_{M} d\right) \xi^{\prime}+\left(x_{2}-x_{0}\right)+\mu_{M}\left(y_{2}-y_{0}\right) .
\end{aligned}
$$

$\xi_{i}(\mathrm{i}=1,2,3)$ are the local coordinates of the collocation points on the element. With a linear transformation $\zeta=\xi-\xi^{\prime}$, eqn (8) can be transformed into

$$
\begin{aligned}
& \int_{\Gamma_{e}} \frac{\phi_{1}-\phi_{10}}{\chi_{M}+\chi_{M}^{0}} d \chi_{M} \\
& \quad=\int_{\Gamma_{e}} \frac{\left[\mu_{M}\left(c \zeta+c \xi^{\prime}+d\right)+\left(a \zeta+a \xi^{\prime}+b\right)\right]\left[\zeta^{2}+\zeta\left(2 \xi^{\prime}-\xi_{3}\right)\right]}{\left(\xi_{1}-\xi_{3}\right) \xi_{1}\left(a_{M} \zeta^{2}+b_{M} \zeta+c_{M}\right)} d \zeta .
\end{aligned}
$$

Then nonlinear transformation $\zeta=\varsigma^{m}$ proposed by Luo et al. [4] can be added to eqn (10), so that it can be calculated using the standard Gaussian quadrature formula.

For $J_{M}^{\prime}$, second Taylor series are essential, and the equation can be separated into three parts as

$$
\begin{gathered}
J_{M}^{\prime}=\int_{\Gamma_{e}} \frac{\phi_{q}-\left(\phi_{q 0}+\phi_{q 0}^{\prime} \chi_{M}\right)}{\left(\chi_{M}+\chi_{M}^{0}\right)^{2}} d \chi_{M}+\int_{\Gamma_{e}} \frac{\phi_{q 0}-\phi_{q 0}^{\prime} \chi_{M}^{0}}{\left(\chi_{M}+\chi_{M}^{0}\right)^{2}} d \chi_{M} \\
\quad+\int_{\Gamma_{e}} \frac{\phi_{q 0}^{\prime}}{\chi_{M}+\chi_{M}^{0}} d \chi_{M} .
\end{gathered}
$$

The third part can be evaluated as in eqn (7), and the second part can also be evaluated by

$$
\int_{\Gamma_{e}}\left[1 /\left(\chi_{M}+\chi_{M}^{0}\right)^{2}\right] d \chi_{M}=-\left.\left[1 /\left(\chi_{M}+\chi_{M}^{0}\right)\right]\right|_{\Gamma_{e}} .
$$

With linear transformation, the first part when $q=1$ can be transformed into

$$
\begin{aligned}
& \int_{\Gamma_{e}} \frac{\phi_{1}-\left(\phi_{10}+\phi_{10}^{\prime} \chi_{M}\right)}{\left(\chi_{M}+\chi_{M}^{0}\right)^{2}} d \chi_{M} \\
& \quad=\int_{\Gamma_{e}} \frac{\left[\mu_{M}(c \xi+d)+(a \xi+b)\right]\left[1-\left(2 \xi^{\prime}-\xi_{3}\right) a_{M} / b_{M}\right] \zeta^{2}}{\left(\xi_{1}-\xi_{3}\right) \xi_{1}\left(a_{M} \zeta^{2}+b_{M} \zeta+c_{M}\right)^{2}} d \zeta .
\end{aligned}
$$


Nonlinear transformation can also be applied. When $q=2,3$, the formulations of $J_{M}$ and $J_{M}^{\prime}$ are similar, so the integrals can be evaluated in the same way.

For $J_{M}^{\prime \prime}$, it can be changed into

$$
\begin{aligned}
J_{M}^{\prime \prime} & =\int_{\Gamma_{e}} \frac{1}{\chi_{M}+\chi_{M}^{0}} \psi_{q} d \chi_{M} \\
\psi_{q} & =\frac{\mu_{M} n_{1}\left(\boldsymbol{x}_{0}\right)-n_{2}\left(\boldsymbol{x}_{0}\right)}{\mu_{M} n_{1}(\boldsymbol{x})-n_{2}(\boldsymbol{x})} \phi_{q} .
\end{aligned}
$$

which has a similar form to $J_{M}$, so it can be evaluated similarly.

\subsection{Straight element}

For isoparametric quadratic element, the denominators of the nearly singular integrals possess a relative high order of $\xi$, which brings difficulty for the integration. So the non-isoparametric quadratic straight line element is introduced. With the straight line element applied, the nearly singular integrals can be integrated analytically, and the separated parts have simple expressions. The boundary quantities are described by the same shape functions as the isoparametric quadratic element. $z_{M}-z_{M}^{0}$ can be represented by a linear function of $\xi$

$$
z_{M}-z_{M}^{0}=\left(a+\mu_{M} c\right) \xi+\left(b+\mu_{M} d\right)-\left(x_{0}+\mu_{M} y_{0}\right)
$$

where

$$
\begin{aligned}
& a=\left(x_{3}-x_{1}\right) / 2, \\
& b=\left(x_{1}+x_{3}\right) / 2, \\
& c=\left(y_{3}-y_{1}\right) / 2, \\
& d=\left(y_{1}+y_{3}\right) / 2 .
\end{aligned}
$$

$\left(x_{1}, y_{1}\right)$ and $\left(x_{3}, y_{3}\right)$ are respectively the start and end point of the element. So $\xi$ gains the following expression

$$
\xi=\frac{z_{M}-z_{M}^{0}-\left[b-x_{0}+\mu_{M}\left(d-y_{0}\right)\right]}{a+\mu_{M} c} .
$$

Nearly singular integrals can be expressed as

$$
\begin{aligned}
L_{M} & =\int_{\Gamma_{e}} \frac{\phi_{q}}{z_{M}-z_{M}^{0}} d z_{M}, \\
L_{M}^{\prime} & =\int_{\Gamma_{e}} \frac{\phi_{q}}{\left(z_{M}-z_{M}^{0}\right)^{2}} d z_{M}, \\
L_{M}^{\prime \prime} & =\frac{\left[\mu_{M} n_{1}\left(\boldsymbol{x}_{0}\right)-n_{2}\left(\boldsymbol{x}_{0}\right)\right] \sqrt{a^{2}+c^{2}}}{A_{M}} L_{M} .
\end{aligned}
$$

So, only $L_{M}$ and $L_{M}^{\prime}$ should be evaluated. 
Let $q=1$, with the substitution of eqn (16) into $\phi_{1}$, the shape function can be expressed as

$$
\phi_{1}=\frac{\left(z_{M}-z_{M}^{0}\right)^{2}-\left[2 B_{M}+\left(\xi_{2}+\xi_{3}\right) A_{M}\right]\left(z_{M}-z_{M}^{0}\right)+\left(B_{M}+\xi_{2} A_{M}\right)\left(B_{M}+\xi_{3} A_{M}\right)}{A_{M}^{2}\left(\xi_{1}-\xi_{2}\right)\left(\xi_{1}-\xi_{3}\right)}
$$

where $A_{M}=a+\mu_{M} c, \quad B_{M}=b-x_{0}+\mu_{M}\left(d-y_{0}\right)$. Therefore $L_{M}$ can be separated into three parts as follows

$$
\begin{aligned}
\int_{\Gamma_{e}} \frac{\phi_{1}}{z_{M}-z_{M}^{0}} d z_{M} & =\frac{1}{\left(\xi_{1}-\xi_{2}\right)\left(\xi_{1}-\xi_{3}\right) A_{M}^{2}} \int_{\Gamma_{e}}\left(z_{M}-z_{M}^{0}\right) d z_{M} \\
& -\frac{2 B_{M}+\left(\xi_{2}+\xi_{3}\right) A_{M}}{\left(\xi_{1}-\xi_{2}\right)\left(\xi_{1}-\xi_{3}\right) A_{M}^{2}} \int_{\Gamma_{e}} d z_{M} \\
+ & \frac{\left(B_{M}+\xi_{2} A_{M}\right)\left(B_{M}+\xi_{3} A_{M}\right)}{\left(\xi_{1}-\xi_{2}\right)\left(\xi_{1}-\xi_{3}\right) A_{M}^{2}} \int_{\Gamma_{e}} \frac{1}{z_{M}-z_{M}^{0}} d z_{M} .
\end{aligned}
$$

The three integrals can be evaluated analytically

$$
\begin{gathered}
\int_{\Gamma_{e}}\left(z_{M}-z_{M}^{0}\right) d z_{M}=\left.\left[\left(z_{M}-z_{M}^{0}\right)^{2} / 2\right]\right|_{\Gamma_{e}} . \\
\int_{\Gamma_{e}} d z_{M}=\left.z_{M}\right|_{\Gamma_{e}}, \int_{\Gamma_{e}}\left[1 /\left(z_{M}-z_{M}^{0}\right)\right] d z_{M}=\left.\log \left(z_{M}-z_{M}^{0}\right)\right|_{\Gamma_{e}} .
\end{gathered}
$$

With similarity of the expressions, the integrals can be evaluated in the same way when $q=2,3$.

Similarly, $L_{M}^{\prime}$ can be also divided into three parts. Two of them can be integrated by eqn .(22), the other one can be evaluated as follows

$$
\int_{\Gamma_{e}}\left[1 /\left(z_{M}-z_{M}^{0}\right)^{2}\right] d z_{M}=-\left.\left[1 /\left(z_{M}-z_{M}^{0}\right)\right]\right|_{\Gamma_{e}} .
$$

\section{Numerical examples}

\subsection{A central crack in finite plate}

For the first example, a central crack of length $2 \mathrm{a}$ in a homogeneous and linear PZT-4 piezoelectric plate is considered to verify the correctness of the two methods. As shown in Figure 1, the plate with a width of $2 \mathrm{~L}$ and height of $2 \mathrm{H}$ is under a pure uniform mechanical tensile loading of $\sigma_{0}=1 \mathrm{Mpa}$.

Normalized stress intensity factor $K_{I} /\left(\sigma_{0} \sqrt{\pi a}\right)$ is presented versus L/a of the finite plate, the results have been plotted in Figure 2 and compared with the corresponding FEM results presented by Cao and Kuang [5] to test the accuracy of the present integration methods. Different heights are considered with $\mathrm{H} / a=0.368,0.568,0.968$ and 4.618 . The two methods coincide well with each other, and they also match well with the FEM results. It should be pointed out that the FEM results are obtained for the condition that the thickness $b$ of the plate is $0.01 a$. 




Figure 1: A central crack in a piezoelectric plate.

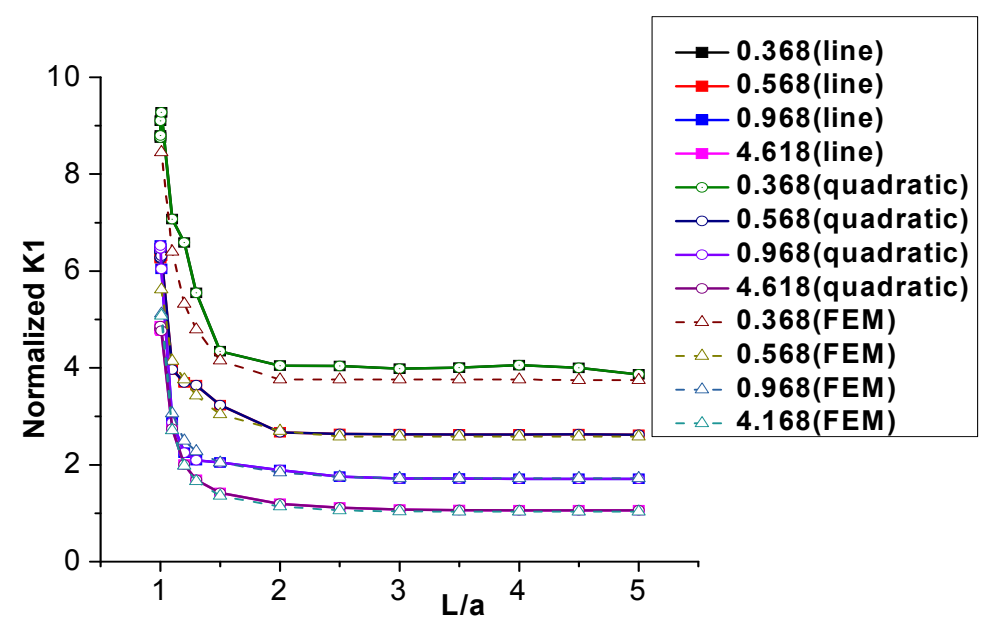

Figure 2: $\quad$ Normalized stress intensity factors for different dimensions.

\subsection{A central crack in thin piezoelectric structure}

When $\mathrm{H}$ decreases to a very small value, the plate becomes piezoelectric film. Since the film is thin, the crack length is set to be relatively small. Let $\mathrm{L} / \mathrm{a}=25$, 50 , normalized stress intensity factor is presented versus $\mathrm{H} / \mathrm{a}$ which is changing from 1 to $1 \times 10^{-6}$ in Figure 3. When the ratio is as small as $10^{-6}$, it is sufficient for modeling many thin piezoelectric films used in smart materials and microelectro-mechanical systems.

It can be found in Figure 3 that the normalized mode-I SIF $K_{1}$ keeps increasing with the decrease of the ratio $\mathrm{H} / \mathrm{a}$ till $\mathrm{H} / \mathrm{a}$ reaches 0.01 . When $\mathrm{H} / \mathrm{a}$ is less than $0.01, \mathrm{~K}_{1}$ becomes unstable. Since the crack is small, the value of $\mathrm{K}_{1}$ is 
not so high. For the straight line element method, the stress intensity factors at $\mathrm{H} / \mathrm{a}=1 \times 10^{-6}$ jump slightly.

Dynamic intensity factor is also taken into consideration. For $\mathrm{H} / \mathrm{a}=0.1$, $\mathrm{L} / \mathrm{a}=25$, Figure 4 shows the normalized dynamic intensity factor versus dimensionless time $t c_{L} / \mathrm{H}$, where

$$
c_{L}=\sqrt{\left(C_{22}+e_{22}^{2} / \varepsilon_{22}\right) / \rho} \text {. }
$$

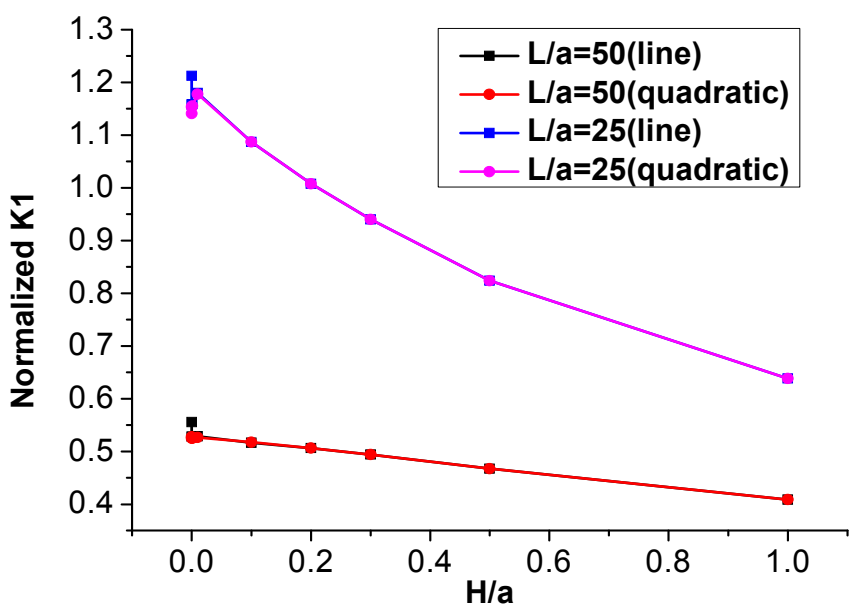

Figure 3: $\quad$ Normalized stress intensity factor versus H/a.

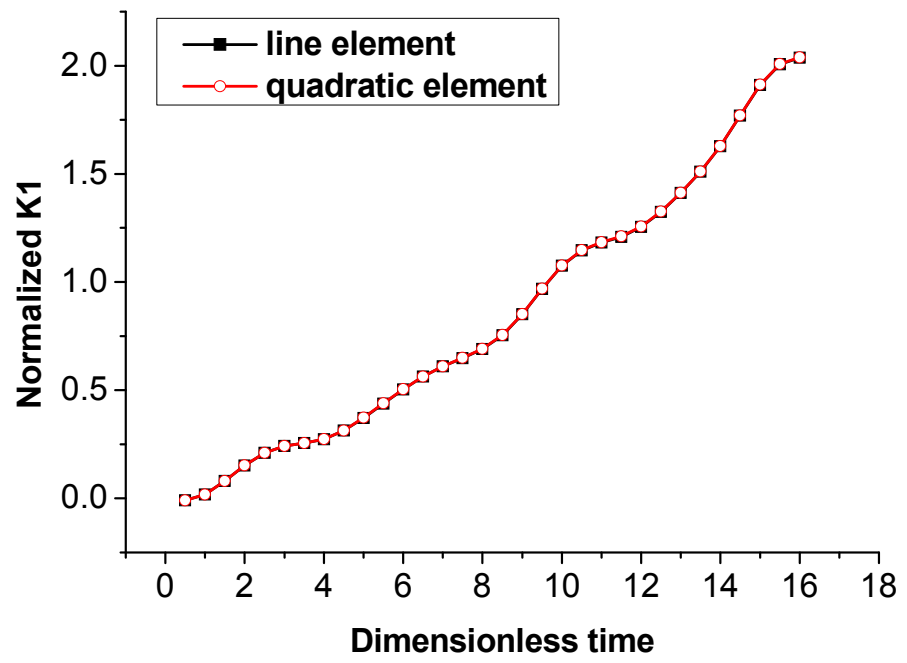

Figure 4: Normalized dynamic stress intensity factors versus dimensionless time. 
The methods of line element and quadratic element are both used and the results coincide with each other very well.

\section{Conclusion}

Static and dynamic crack problems in piezoelectric thin structure are considered. Two methods are presented to deal with the nearly singular problems in the Laplace domain fundamental solutions. The first method for quadratic element is semi-analytical and the second method for straight line element is analytical. Static stress intensity factor of the piezoelectric plate is obtained for different structural dimensions. The results have been compared with FEM results and the agreement verifies the accuracy of the present BEM. Then the crack in thin structure is discussed, normalized intensity factors of both static and dynamic cases are obtained. The results indicate that the two methods coincide well when the ratio of the film thickness to the crack length is as small as $10^{-6}$, which is sufficient for modelling many thin piezoelectric films and coatings.

\section{References}

[1] García-Sánchez, F., Zhang, C.H. and Sáez, A., 2-D transient dynamic analysis of cracked piezoelectric solids by a time-domain BEM. Comput. Methods Appl. Mech. Engrg., 197, pp. 3108-3121, 2008.

[2] Liu, Y.J. and Fan, H., Analysis of thin piezoelectric solids by the boundary element method. Comput. Methods Appl. Mech. Engrg., 191, pp. 22972315, 2002.

[3] Wang, C.Y. and Zhang, C.H., 3-D and 2-D dynamic Green's functions and time-domain BIEs for piezoelectric solid. Engineering Analysis with Boundary Elements, 29, pp. 454-465, 2005.

[4] Luo, J.F., Liu, Y.J. and Berger, E.J., Analysis of two-dimensional thin structures (from micro- to nano-scales) using the boundary element method. Computational Mechanics, 22, pp. 404-412, 1998.

[5] Cao, Z.J. and Kuang, Z.B., A finite element modeling for directly determining intensity factors of piezoelectric materials with cracks. Int $\mathbf{J}$ Fract, 149, pp. 67-85, 2008. 